Check for updates

Cite this: J. Anal. At. Spectrom., 2017, 32, 996

Received 27th January 2017

Accepted 23rd March 2017

DOI: 10.1039/c7ja00039a

rsc.li/jaas

\section{Determination of the platinum concentration of a Pt/silica nanocomposite decorated with ultra small Pt nanoparticles using single particle inductively coupled plasma mass spectrometry}

\author{
András Sápi, ${ }^{a}$ Albert Kéri, ${ }^{b}$ Ildikó Kálomista, ${ }^{b}$ Dorina G. Dobó, ${ }^{a}$ Ákos Szamosvölgyi, ${ }^{a}$ \\ Koppány L. Juhász, ${ }^{a}$ Ákos Kukovecz, ${ }^{\text {ac }}$ Zoltán Kónya (D) ad and Gábor Galbács (D) *b
}

In this study, the performance of five analytical techniques applicable to the determination of the load concentration of ultra small nanoparticles in a $\mathrm{Pt} / \mathrm{SiO}_{2}$ nanocomposite was critically evaluated. Four of the techniques (SEM-EDS, TEM imaging, XPS and solution-mode ICP-MS) are often used for the characterization of nanoparticles, whereas single particle ICP-MS (spICP-MS) is an upcoming, novel methodology. After experimentally testing and discussing the pros and cons of each analytical technique, it was found that spICP-MS is one of the most accurate, precise and practical techniques for the analysis of nanocomposites. This technique works directly with dispersions, the measurement only takes a few minutes, it gives highly reliable results, largely free from interference from precursor residues and can also provide additional information about the particles. Although the individual measurement of ultra small nanoparticles is not yet possible by spICP-MS, the cumulative signal from such load particles in a nanocomposite allows the accurate determination of the load concentration. The spICP-MS result was concordant with the result obtained by TEM imaging, whereas SEM-EDS, XPS and solution-mode ICPMS strongly overestimated the concentration.

\section{Introduction}

Novel metal/metal-oxide nanocomposites are in the focus of intense fundamental research, not only due to their unique characteristics, but also because of their already found several industrial uses in the area of medicine, sensors, energy storage, structural materials or as heterogeneous catalysts for the processing of chemicals or environmental pollutants. The reliable and quick characterization of the synthesized nanomaterials is obviously crucial both in terms of morphology and structure and composition. One of the key parameters assessed is the relative concentration (wt\%) of the active metal and the supporting metal-oxide nanoparticle, as this has significant influence on the activity, selectivity and performance, as was shown e.g. in heterogeneous catalysis,,$^{\mathbf{1}, 2}$ sensors $^{3}{ }^{\text {electrochemical }}{ }^{4}$ or fuel cell ${ }^{5}$ research. For the determination of the concentration of the active metallic component, solution-mode inductively

${ }^{a}$ Department of Applied and Environmental Chemistry, University of Szeged, Rerrich Béla Square 1, 6720 Szeged, Hungary

${ }^{b}$ Department of Inorganic and Analytical Chemistry, University of Szeged, Dóm Square 7,6720 Szeged, Hungary. E-mail: galbx@chem.u-szeged.hu

"MTA-SZTE "Lendület” Porous Nanocomposites Research Group, Rerrich Béla Square 1, H-6720 Szeged, Hungary

${ }^{d}$ MTA-SZTE Reaction Kinetics and Surface Chemistry Research Group, Rerrich Béla Square 1, H-6720 Szeged, Hungary coupled plasma mass spectrometry (ICP-MS), ${ }^{6-8}$ scanning electron microscopy coupled with energy dispersive spectroscopy $(\mathrm{SEM}-\mathrm{EDS})^{\mathbf{9}, 10}$ or X-ray photoelectron spectroscopy (XPS) ${ }^{\mathbf{1 1}}$ is typically used in the literature.

Single particle, or particle-mode, ICP-MS (spICP-MS) is a novel nanoparticle (NP) characterization technique, which is recently gaining increasing attention in the literature owing to its versatility and high throughput. ${ }^{\mathbf{1 2 - 1 5}}$ In this technique, sensitive ICP-MS spectrometers are used in the time-resolved mode for the measurement of dilute nanodispersions (particle concentrations less than $10^{5} \mathrm{~mL}^{-1}$ are adequate). The nanoparticles get atomized and ionized in the plasma. The signal from one of the constituting elements is monitored as a function of time, thus each detected NP will produce a narrow signal peak (a few hundred $\mu$ s duration), the height of which is proportional to the mass of the analyte present in the NP. After the statistical evaluation of the signal time profile and assuming a spherical NP geometry, information can be obtained about not only the elemental (isotopic) composition of the NPs, but also their characteristic size and distribution, as well as the particle concentration. The measurement is fast (takes only a couple of minutes) and the required sample volume is very small. For metallic NPs, size detection limits ranging from 10 to $30 \mathrm{~nm}$ and upper detectable size limits around or above a few hundred $\mathrm{nm}$ are typically reported. ${ }^{\mathbf{1 6 - 1 9}}$ This technique is 
nowadays considered to be a faster and more practical alternative to other established NP characterization methods such as SEM or DLS. ${ }^{20}$

The goal of the present study was to assess the performance of the novel spICP-MS technique in an application challenging for this technique: the determination of the concentration of $\mathrm{Pt}$ in a Pt/silica nanocomposite newly synthesized for the purpose of this study. In this nanocomposite, the active ultra small Pt NPs are anchored to the surface of the support, the ratio of the diameter of the two particles is over 200, and the loading is low $(<1 \mathrm{wt} \%)$. A critical performance comparison is provided with other well established NP characterization methods, such as transmission electron microscopy (TEM), SEM-EDS, solutionmode ICP-MS and XPS.

\section{Experimental section}

\subsection{Synthesis and morphology of the particles}

Synthesis of Stöber silica particles. Silica particles were prepared through a process based on the Stöber method, ${ }^{28}$ using tetraethoxysilane (TEOS) as the precursor. The synthesis was carried out by mixing an ethanolic TEOS solution $(17 \mathrm{~mL}$ TEOS dissolved in $200 \mathrm{~mL}$ ethanol) and an ethanolic ammonia solution $(100 \mathrm{~mL}$ ammonia-solution of $25 \mathrm{wt} \%$ concentration mixed with $70 \mathrm{~mL}$ distilled water and $110 \mathrm{~mL}$ ethanol). The obtained dispersion was then stirred for $24 \mathrm{~h}$. The precipitate was washed and collected by centrifugation and then finally dried at $60{ }^{\circ} \mathrm{C}$ for $12 \mathrm{~h}$. Individual spheres with a size of $449 \pm$ $76 \mathrm{~nm}$, as measured by SEM, were obtained, however a small amount of aggregates of 2-3 spheres was also observed.

Synthesis of ultra small Pt nanoparticles. For the synthesis of ultra small Pt NPs, $29 \mathrm{mg}$ of $\mathrm{PtCl}_{4}$ and $50 \mathrm{mg}$ of $\mathrm{NaOH}$ were dissolved in two separate portions of $2.5 \mathrm{~mL}$ ethylene glycol. The solutions were then mixed and heated to $160{ }^{\circ} \mathrm{C}$. The temperature of the mixture was held constant for 3 hours under an Ar atmosphere. After cooling, $2.5 \mathrm{~mL}$ of a $1 \mathrm{M} \mathrm{HCl}$ solution was added to the black suspension and the resulting NPs were collected by centrifugation. The NPs were re-dispersed in $10 \mathrm{~mL}$ of $2.1 \mathrm{mg} \mathrm{mL}{ }^{-1}$ polyvinylpyrrolidone (PVP, $M_{\mathrm{w}}=40.000$ ) in ethanol with ultrasonication. The NPs were finally washed by repeatedly performing hexane precipitation/ethanol redispersion cycles. The morphology of the Pt NPs was studied by TEM and they were found to be spherical with a narrow size distribution $(1.6 \pm 0.5 \mathrm{~nm})$. The electron diffraction pattern showed the presence of the face-centered cubic (fcc) phase of the metallic platinum.

Synthesis of Pt nanoparticles. The larger Pt NPs used in the spICP-MS size calibration process were synthesized by a multistep seed-mediated method described by Bigall et al. ${ }^{29}$ For the preparation of seed particles, $\mathrm{H}_{2} \mathrm{PtCl}_{6} \cdot 6 \mathrm{H}_{2} \mathrm{O}$ solution ( $0.2 \mathrm{wt} \%$ ) was added to $400 \mathrm{~mL}$ boiling distilled water under continuous stirring. The next step was the addition of a $10 \mathrm{~mL}$ solution of Na-citrate $(1 \%)$ and citric acid $(0.05 \%)$ buffer, followed by homogenization and the addition of $5 \mathrm{~mL} 0.08 \% \mathrm{NaBH}_{4}$ in the above-mentioned Na-citrate-citric acid buffer. The suspension was then refluxed for 10 minutes and cooled down to room temperature. The seed particles produced by using this procedure were used for the preparation of $20 \mathrm{~nm}$ particles via the further addition of the appropriate amount of $\mathrm{H}_{2} \mathrm{PtCl}_{6}$ $\cdot 6 \mathrm{H}_{2} \mathrm{O}$ solution of the Na-citrate-ascorbic acid buffer. During the preparation of larger particles, the same procedure was used as mentioned above except that the smaller particles were used as seeds (e.g. the $20 \mathrm{~nm}$ particles seeded the $40 \mathrm{~nm}$ particles, and so on). Finally, the characteristic size of all large NPs was determined by TEM and dynamic light scattering (DLS). They were found to have characteristic diameters of $20.1 \mathrm{~nm}$, $40.7 \mathrm{~nm}, 51.5 \mathrm{~nm}$ and $58.2 \mathrm{~nm}$.

Preparation of the $\mathbf{P t} / \mathrm{SiO}_{2}$ nanocomposite particles. The $1.6 \mathrm{~nm}$ Pt NPs and the Stöber silica particles were mixed together in ethanol and sonicated in an ultrasonic bath $(40 \mathrm{kHz}$, $80 \mathrm{~W}$ ) for 3 hours. The nanocomposite particles were then collected by centrifugation. The products were washed with ethanol three times before they were dried at $80{ }^{\circ} \mathrm{C}$ overnight. Beside homogeneously distributed Pt NPs, small islands of separate particles were also observed by TEM on the surface of the silica particles. Fig. 1 and its insets show the electron microscopy images of the $1.6 \mathrm{~nm}$ Pt NPs, the silica supported NPs and the $\mathrm{Pt} / \mathrm{SiO}_{2}$ nanocomposite NPs, respectively.

\subsection{Instrumentation and data evaluation}

The morphology of the pure Pt nanoparticles and Stöber silica, as well as the $\mathrm{Pt} /$ silica nanocomposites, was investigated by using a TEM (FEI Tecnai $\mathrm{G}^{2} 20$ X-TWIN operating at $200 \mathrm{kV}$ ) and a SEM (Hitachi S- 4700 operating at $20 \mathrm{kV}$, also equipped with a Röntec energy dispersive spectrometer with a $12 \mathrm{~mm}$ working distance). The crystal structure of the silica was examined by Xray Diffraction (XRD, Rigaku MiniFlex II Desktop Diffractometer operating with a Cu $\mathrm{K}_{\alpha}$ source $(\lambda=0.1542 \mathrm{~nm})$ at $30 \mathrm{kV}$ and 15 $\mathrm{mA})$. The density of the particles was measured by the $\mathrm{N}_{2}$ gas adsorption method using a Quantachrome NOVA 3000e gas sorption instrument.

The concentration of the Pt nanoparticles anchored onto the surface of the Stöber silica spheres in the nanocomposite was determined by using ICP-MS, XPS, SEM-EDS measurements and TEM imaging. The measurement conditions and data evaluation approaches employed are described below in detail.

X-ray photoelectron spectroscopy. X-ray photoelectron spectra were recorded using a SPECS instrument equipped with a PHOIBOS 150 MCD 9 hemispherical electron energy analyzer, using Al $\mathrm{K}_{\alpha}$ radiation $(h \nu=1486.6 \mathrm{eV})$. The X-ray gun was operated at $210 \mathrm{~W}(14 \mathrm{kV}, 15 \mathrm{~mA})$. The analyzer was operated in the FAT mode, with the pass energy set to $20 \mathrm{eV}$. The step size was $25 \mathrm{meV}$ and the collection time in one channel was $250 \mathrm{~ms}$. Typically, 5-10 scans were added up to acquire a single spectrum. Energy referencing was not applied. In all cases the powder-like samples were evenly laid out on one side of a double-sided adhesive tape, the other side being attached to the sample holder of the XPS instrument. The samples were evacuated at room temperature and then inserted into the analysis chamber of the XPS instrument.

Solution-mode inductively coupled plasma mass spectrometry. An Agilent Technologies 7700X ICP-MS instrument was used in all ICP-MS experiments. Sample introduction was 

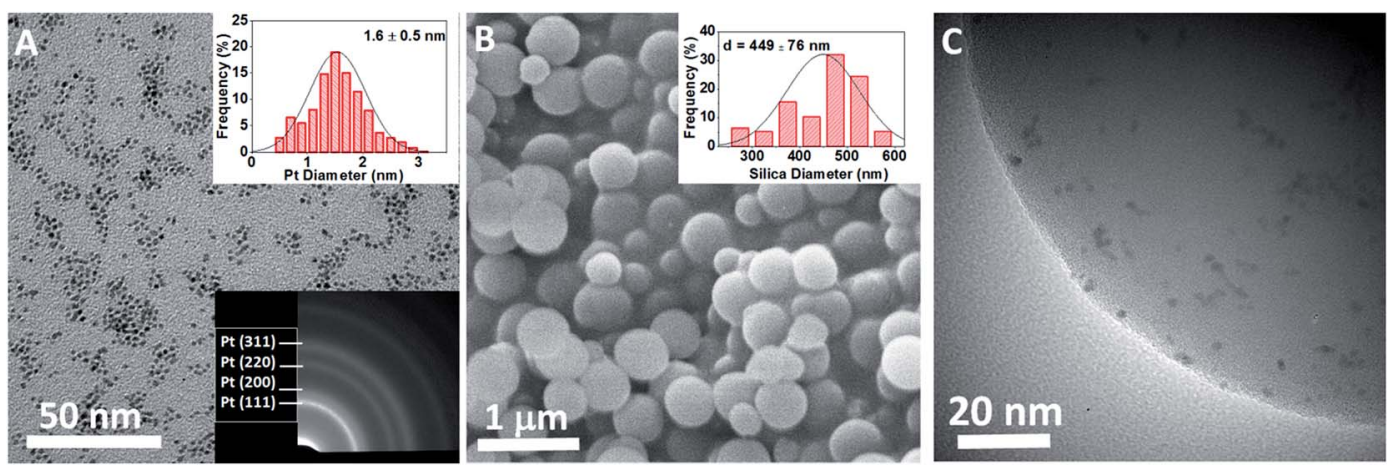

Fig. 1 Typical TEM image of the $1.6 \mathrm{~nm}$ Pt nanoparticles (A), SEM micrograph of the pristine Stöber silica spheres (B) and TEM image of the Pt nanoparticles anchored onto the surface of a Stöber silica sphere (C).

performed by using an Agilent I-AS autosampler and a Micro Mist pneumatic nebulizer equipped with a Peltier-cooled Scotttype spray chamber. The sample uptake rate was $400 \mu \mathrm{L} \mathrm{min}{ }^{-1}$. The ICP plasma and interface parameters were set up according to standard conditions (RF forward power: $1550 \mathrm{~W}$, argon carrier gas flow rate: $1.05 \mathrm{~L} \mathrm{~min}^{-1}$, sampling depth: $8.0 \mathrm{~mm}$ ), and then a fine tuning was performed using solutions supplied by Agilent (No. G1820-60410) and special attention was paid to the optimization of the plasma sampling depth. All measurements were performed by monitoring the signal of the ${ }^{195} \mathrm{Pt}$ isotope. In order to avoid possible interference from $\mathrm{HfO}$ in the multielement solution standard used for calibration, the measurements were performed in He mode of the collision cell. It is also worth considering that even if the HfO interference would increase the Pt signals due to the Hf content of the solution standard, it could only cause a negative error in the Pt concentration result, since the samples contain no Hf. The labware (PE autosampler vials, certified PMP volumetric flasks, etc.) was thoroughly cleaned before use with dilute ultratrace quality hydrochloric and nitric acid. The sample preparation procedure was optimized for the complete dissolution of the $\mathrm{Pt}$ content of the nanocomposite. Several sample dissolution approaches of stepwisely increasing aggressivity and duration (direct dispersion in water, $30 \mathrm{~min}$ sonication in water, $30 \mathrm{~min}$ sonication in $\mathrm{HCl}$, and $30 \mathrm{~min}$ digestion at $130{ }^{\circ} \mathrm{C}$ in $3: 1 \mathrm{HCl}-$ $\mathrm{HNO}_{3}$ ) were tested and the one producing the highest $\mathrm{Pt}$ concentration measurable by solution-mode ICP-MS analysis was chosen as the optimal one (digestion in boiling $3: 1 \mathrm{HCl}-$ $\mathrm{HNO}_{3}$ ). A further increase in the duration of acid treatment (from $30 \mathrm{~min}$ to $120 \mathrm{~min}$ ) resulted in no further significant increase in the Pt concentration measured.

Particle-mode inductively coupled plasma mass spectrometry. For spICP-MS measurements, the ICP-MS instrument was set up as described above for solution-mode measurements, except that the data acquisition software was used in Time Resolved Analysis (TRA) mode, with the integration time set to $10 \mathrm{~ms}$. This relatively long dwell time was chosen in consideration of the large $(c a .450 \mathrm{~nm})$ silica carrier nanoparticle, thereby ensuring that the complete Pt signal peak produced by each nanocomposite particle is collected. All nanodispersions were diluted to less than $10^{4} \mathrm{~mL}^{-1}$ particle concentration, a ten times lower value than typical in spICP-MS, in order to completely avoid any NP signal peak overlaps. Data evaluation in spICP-MS was performed on the histograms (frequencycount diagrams) produced from the time-resolved signal traces, as described in ref. 15. Data processing was performed using Agilent MassHunter (Santa Clara, California, USA) and OriginLab Origin (Northampton, Massachusetts, USA) software.

Transmission electron microscopy imaging. The Pt concentration of the nanocomposite was determined by this method via the counting of the load particles on the surface of the support particles. TEM measurement conditions were as described earlier. Since only a portion of the surface of the large silica support particles could be observed in all micrographs due to the large magnification required for the counting of the ultra small load particles, a simple geometric calculation was performed to extrapolate the data. The radius of all nanocomposite spheres $(R)$ processed was calculated after aligning an arc in the TEM image processing software (ImageJ) to the edge of the circular projection of each individual spheres on the micrographs. Next, a circular segment with a height of $h$ was marked on the circular projection of each sphere by drawing a chord through two separate, distant points on the edge of projection; the area of the corresponding spherical cap can then be calculated by using the formula of $A_{\text {cap }}=R \pi h$. The number of Pt NPs within the area of the spherical cap is two times the number of countable particles on the circular segment $(N)$. The total number of NPs on the surface of a silica sphere can then be estimated by ratioing the two areas:

$$
N_{\text {total }}=\frac{A_{\text {sphere }}}{A_{\text {cap }}} 2 N=\frac{4 R^{2} \pi}{R \pi h} 2 N=\frac{8 R N}{h}
$$

Using the density data of platinum $\left(\rho_{\mathrm{Pt}}\right)$ and silica $\left(\rho_{\text {silica }}\right)$, as well as the radius of the Pt NPs $(r)$, the concentration was finally calculated according to the formula

$$
\text { conc. }=\frac{\rho_{\mathrm{Pt}} V_{\mathrm{Pt}}}{\rho_{\mathrm{Pt}} V_{\mathrm{Pt}}+\rho_{\text {silica }} V_{\text {silica }}}=\frac{\rho_{\mathrm{Pt}} N_{\text {total }} \frac{4 r^{3} \pi}{3}}{\rho_{\mathrm{Pt}} N_{\text {total }} \frac{4 r^{3} \pi}{3}+\rho_{\text {silica }} \frac{4 R^{3} \pi}{3}}
$$


Scanning electron microscopy coupled with energy dispersive spectroscopy. The parameters of the SEM instrumentation used here were described earlier in this section. The powder sample was fixed on a carbon tape attached to the surface of an alumina sample holder. The distance of the detector was $30 \mathrm{~mm}$ and the working distance was $12 \mathrm{~mm}$ during the measurements. The Si drift detector was equipped with a Be window and placed at an angle of $30^{\circ}$. The accelerating voltage was $20 \mathrm{kV}$, the sample current was $10 \mu \mathrm{A}$ and a $100 \mu \mathrm{m}$ size aperture was used. All EDS data were recorded for 20 minutes on each spot. The Pt concentration was calculated using the $\mathrm{Si} \mathrm{K}_{\alpha}$ and the $\mathrm{Pt} \mathrm{L}_{\alpha}$ signals.

\subsection{Chemicals}

All chemicals used in the synthesis experiments (tetraethoxysilane, ethanol, ammonia, $\mathrm{PtCl}_{4}$, ethylene glycol, polyvinylpyrrolidone, $\mathrm{H}_{2} \mathrm{PtCl}_{6} \cdot 6 \mathrm{H}_{2} \mathrm{O}$, Na-citrate, citric acid, $\mathrm{NaBH}_{4}$, and ascorbic acid) were of analytical grade or better quality. Dilution of all solutions and dispersions was carried out with class I (trace analytical) quality deionized water from a VWR Puranity TU 6 UV/UF device. The cleaning of ICP-MS labware, as well as the acid dissolution of Pt NPs anchored to the silica particles solutions, was carried out by using the solution prepared from ultratrace quality nitric (67 wt\%) and hydrochloric (37 wt\%) acids (Ultrapure Normatom, VWR). ICP-MS solution calibration standards were prepared from a certified, multielement stock solution (No. 8500-6948, Agilent Technologies). The argon (99.996\%) technical gas used was obtained from Messer (Hungary).

\section{Results and discussion}

\subsection{Single particle ICP-MS measurements}

The direct, individual measurement of the ultra small Pt NPs anchored onto the surface of the silica particles is not possible by spICP-MS due to the insufficient sensitivity of present day ICP-MS instruments (typical size detection limits for metallic NPs are between 10 and $30 \mathrm{~nm}$ ). The signal pulse from each individual Pt NPs present in a single nanocomposite particle will add up and produce one Pt signal peak per nanocomposite. Now we would like to point out that this cumulative signal can be used for the quantitation of Pt in the nanocomposite, if the Pt concentration is "large" enough. Our calculations show that if the concentration of $\mathrm{Pt}$ is over $0.01-0.02 \mathrm{wt} \%$ in the nanocomposite, then the cumulative $\mathrm{Pt}$ signal should be well detectable by ICP-MS. After a size calibration of the spICP-MS using Pt particle standards, the detected cumulative peak intensity can be converted to the diameter value of an "equivalent" Pt NP and then the mass of Pt present in each nanocomposite particle can be calculated (Fig. 2).

We would also like to support the feasibility of the above proposal in the present application by adding two comments. First, spICP-MS measurements have already been shown to have an excellent repeatability (precision) due to the fact that the characteristic signal (mode of the signal histogram) is based on the measurement of more than ten thousand particles. Better than $1 \%$ RSD values can be achieved even when characteristic pulse intensities as small as 1-10 counts are measured. ${ }^{\mathbf{1 5 , 2 1}}$ This feature allows the accurate and precise measurement of small analyte signals. Second, some considerations can also be given to the fact that in the present challenging application, the supporting silica particles are quite large, near to or even over the dynamic range of spICP-MS, ${ }^{16,22}$ thus a complete ionization of the silica particles may not be achieved in the plasma. Note that however even if the ionization of the support particles would be less than $100 \%$, the Pt NPs anchored onto the surface will surely get completely ionized as the atomization and ionization of the nanocomposite starts on its surface and progresses inwards during the residence of the particle in the plasma.

The above-mentioned concept was successfully tested with the measurement of the present $\mathrm{Pt} / \mathrm{SiO}_{2}$ nanocomposite, as can be seen in Fig. 3. After optimizing ICP-MS conditions with special attention paid to the plasma sampling depth, ${ }^{23}$ size calibration was carried out (Fig. 3b) using the large Pt NPs prepared for this purpose (as described in Section 2.1). After evaluating the data using the three sigma methodology, ${ }^{19,24}$ we established that the Pt size detection limit is $17.2 \mathrm{~nm}$.

Fig. 3a shows that the lognormal Pt NP peak in the signal histogram is well resolved from the background peak (caused by the spectral background and any residual dissolved $\mathrm{Pt}$ ). The lognormal fit is of good quality and is based on the analysis of thousands of nanocomposite particles. The mode of the particle peak (characteristic NP signal) was found to be equivalent to the signal from a $20.4 \mathrm{~nm}$ diameter spherical Pt particle. Knowing the size and density of both the support and the load particles, the $\mathrm{Pt} w \mathrm{w} \%$ concentration was calculated. This calculation produced a value of $0.0966 \mathrm{wt} \%$, with a standard deviation of 0.0025 based on three repeated measurements.

\subsection{Discussion of the accuracy of Pt concentration data}

In addition to spICP-MS, the determination of the concentration of the Pt nanoparticles on the surface of the silica support was also carried out by four other analytical methods, namely solution-mode ICP-MS, SEM-EDS, TEM and XPS. The obtained results can be seen from Table 1 . Three parallel measurements were performed with every method in order to calculate the standard deviation.

As it can be seen from the table, the concentration results are significantly different. TEM and spICP-MS results agree reasonably well, but the concentrations provided by the other three methods are several times higher. In view of these differences, it is beneficial to review the theoretical limitations of each individual analytical method in this application.

The analysis by the TEM imaging method is fairly simple to execute and requires only a small amount of solid sample. It is also very sensitive as very small (even sub $\mathrm{nm}$ ) NPs can be counted. At the same time, the calculation relies on a number of extrapolations and preconditions including the following: (a) due to the large magnification needed to resolve the ultra small load NPs, only a portion of the total surface of the support particle is imaged, (b) the surface coverage is assumed to be the same on all sides of the support particle, (c) the surface coverage should not be too large to avoid clustering, (d) the NPs found on 


\section{PLASMA (IONIZATION) ZONE}
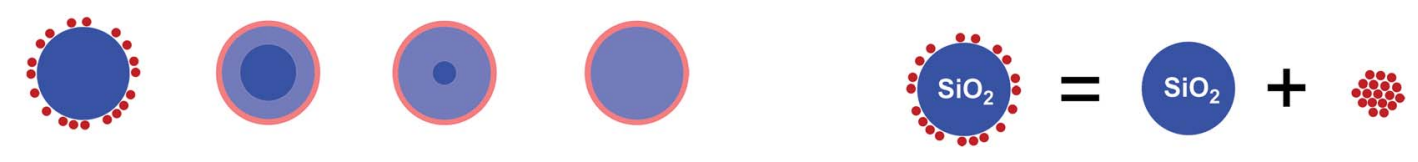

\section{residence time}

Fig. 2 Left panel: schematic illustration of the decomposition and ionization process that a nanocomposite particle undergoes in the ICP plasma (deep blue: solid silica particle, light blue: Si and O ion cloud, deep red: surfacial solid Pt NPs, light red: Pt ion cloud). Right panel: if the concentration is large enough, the ultra small Pt NPs produce a cumulative signal, equivalent to that of a detectable larger NP.
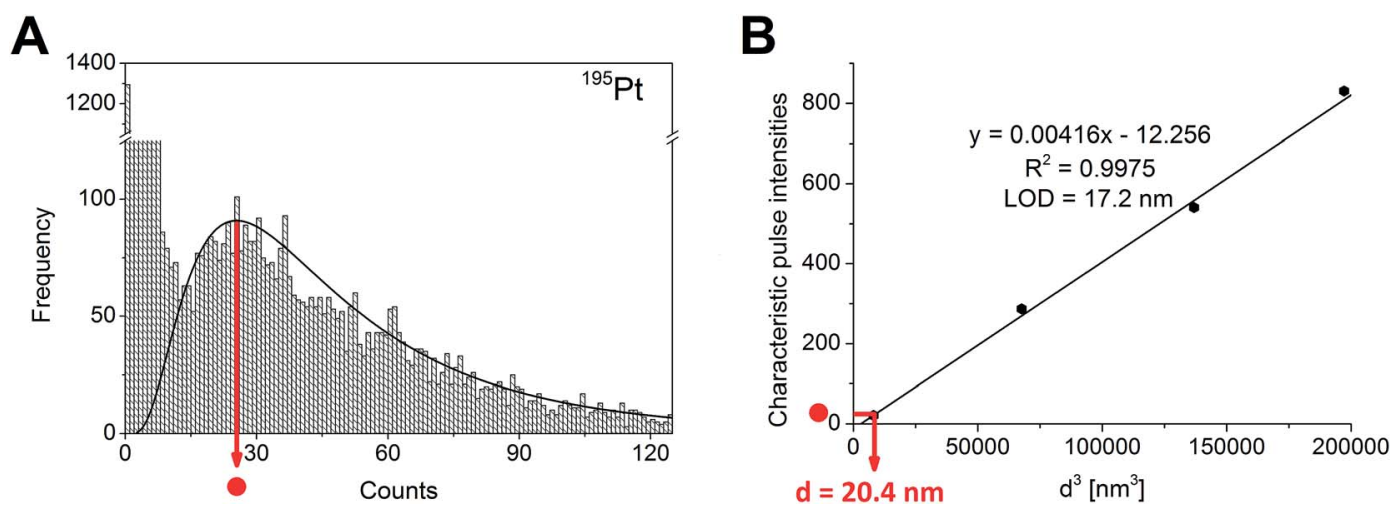

Fig. $3{ }^{195} \mathrm{Pt}$ spICP-MS signal histogram recorded for the $\mathrm{Pt} / \mathrm{SiO}_{2}$ nanocomposite (left panel). Please note that the lognormal NP signal peak is well separated from the solution background signal. The characteristic intensity of the NP peak in the histogram corresponds to an equivalent NP size of $20.4 \mathrm{~nm}$ in the spICP-MS size calibration curve obtained with pure Pt NPs (right panel).

the surface can only be assumed to be of the quality hoped (here: $\mathrm{Pt}$ ), since there is no direct information about the composition of the load NPs, and (e) the load NPs are assumed to be completely spherical, which is an assumption that rarely holds for very small NPs. In addition to this, the sample preparation/vacuum introduction as well as the processing of some dozens of images (the maximum in routine TEM practice) requires a considerable amount of time (hours). A bonus of the approach is that TEM also provides morphological information and can be universally applied to just any active NPs (at least under ultra clean conditions, so that assumption (d) can hold). Overall, the TEM imaging analysis is lengthy, and can provide a concentration

Table 1 Concentration of the Pt nanoparticles in the $\mathrm{Pt} / \mathrm{SiO}_{2}$ nanocomposites determined by the five techniques. Standard deviation values are based on three parallel measurements

\begin{tabular}{lll}
\hline Technique & $\begin{array}{l}\text { Pt concentration } \\
(\text { wt\% })\end{array}$ & Standard deviation \\
\hline Particle-mode ICP-MS & 0.0966 & 0.0025 \\
TEM & 0.0824 & 0.0261 \\
Solution-mode ICP-MS & 0.2821 & 0.0030 \\
SEM-EDS & 0.4400 & 0.0152 \\
XPS & 0.7173 & 0.2867
\end{tabular}

result that is fairly accurate, but not very precise (e.g. consider that the TEM result in Table 1 also has an RSD of over $30 \%$ ).

spICP-MS requires a very small amount of dispersion for the analysis. The measurement is fast and simple, and usually takes only $c a .5$ minutes, although it also requires the availability and measurement of size calibration samples (Pt). The nanocomposite particles decompose in the ICP plasma and the Pt signals from the particles and from the precursor residues (solution background) are clearly differentiated, therefore the quantitative result is accurate and gives the true, nanocomposite-related Pt concentration. This is so because the dissolved analyte content of nanodispersions gives a peak following the Poisson function in the TRA histogram, whereas NPs give a separate peak that follows the lognormal statistics. ${ }^{16,24}$ This can help in avoiding large inaccuracies in quantitative NP analysis. The analysis is also very precise, since it is based on the measurement of thousands of particles. The spICP-MS result obtained in the present study agrees well with the TEM result, and it is also the most precise one out of the five methods tested. A further benefit of spICP-MS analysis is that the technique is also capable of delivering a number of additional, valuable information about the dispersion sample, such as the number concentration and purity of the particles, as well as the presence of precursor residues. 


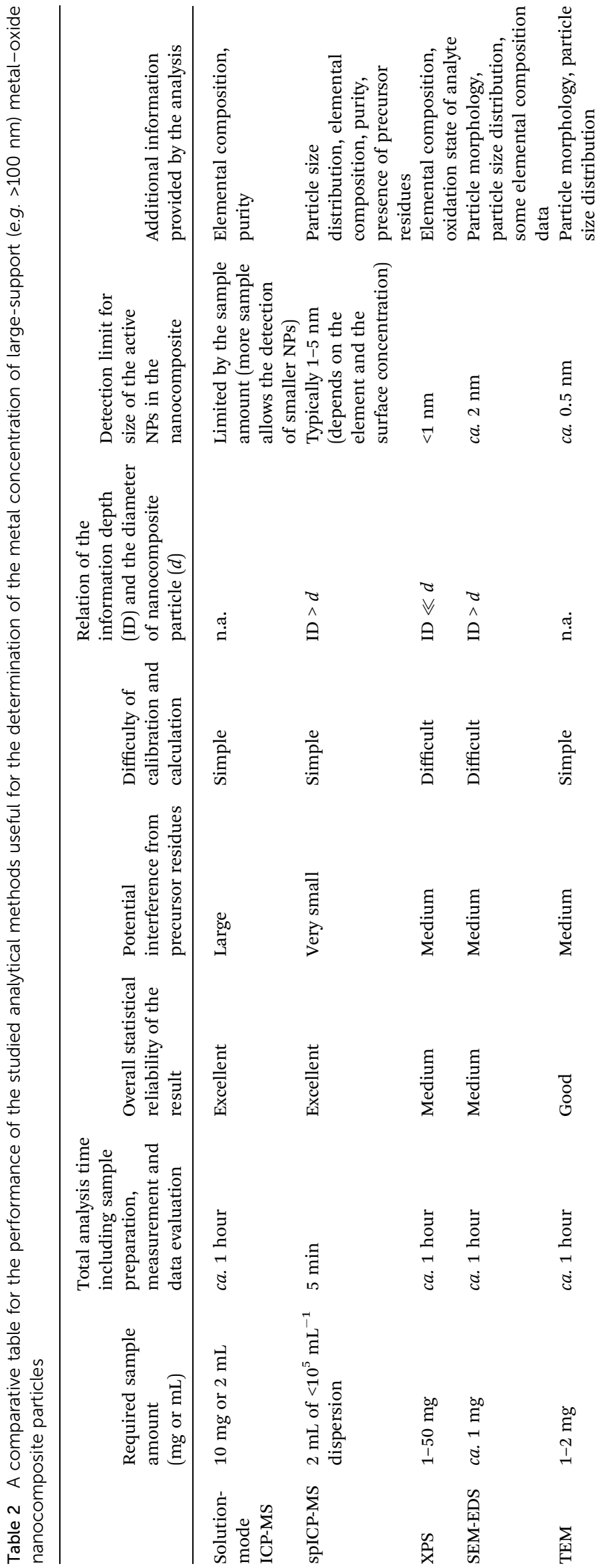


Under the conditions used (e.g. acceleration voltage, working distance, angle of the detector, atomic mass etc.) the SEM-EDS technique has a 5-7 $\mu \mathrm{m}$ information depth according to the Castaing's formula. ${ }^{25}$ Usually, the matrix correction (ZAF correction) is needed for the exact concentration determination due to the fact that the number of X-ray photons counted at the detector depends on the atomic number of the elements, the adsorption of the X-rays and the fluorescence effect. The sensitivity of SEM-EDS analysis is mediocre; the limit of detection values is around a few thousand ppm (e.g. $0.2-0.3 \mathrm{wt} \%),{ }^{26}$ meaning that in the present application, the technique was operated around its limit of detection. In addition to this, the accuracy is best if the sample has a smooth, polished surface (e.g. bulk sample), but in the case of particulate samples, the signal contribution from the "top" and "bottom" surfaces of the particles will not be the same, thus SEM-EDS requires a very careful calibration for quantitative measurements. Consequently, accurate results under routine conditions cannot be expected; here we found that the Pt content reported by SEMEDS is $c a$. four times higher than the result from TEM imaging calculations. The best spatial resolution of EDS is about $1 \mu \mathrm{m}$, which might allow for the individual analysis of each $\mathrm{Pt} / \mathrm{SiO}_{2}$ nanocomposite if the particles are well separated on the substrate, but in order to have a larger analytical signal, typically larger apertures are used (we used $100 \mu \mathrm{m}$ ). This brings about the problem of potential interference from precursor residues which can contaminate the substrate in areas between the nanocomposite particles during sample preparation. This effect can result in a significant positive bias in the SEM-EDS results. SEM-EDS requires only a very small amount of solid sample for the analysis. The analysis time is long ( $\geq$ one hour), similar to TEM imaging, considering sample preparation, vacuum introduction and the need for longer exposition times when working at low concentrations, as well as the complicated data processing. In summary, SEM-EDS is not very suitable for the accurate analysis of nanocomposites with large support particles and/or low loadings.

XPS is a truly surface analytical technique; its information depth is about $10 \mathrm{~nm}$ only. It is somewhat more sensitive than SEM-EDS (a factor of 2-3), but the spatial resolution is poorer (some tens of $\mu \mathrm{m}) .{ }^{26}$ This again permits interference from precursor residues on the substrate, which may result in a positive error in quantitative measurements. Due to its shallow information depth and because of geometric conditions, XPS is not able to detect the majority of the mass of the support particle, which causes a strong overestimation of the wt\% Pt concentration. This can explain the nearly 9 times higher XPS result compared to TEM. The precision was also found to be poor, as much as $40 \%$ RSD. The analysis time is comparable to that of TEM or SEM-EDS ( $\geq$ one hour).

Solution-mode ICP-MS is a very sensitive technique. Its ng $\mathrm{L}^{-1}$ (ppt) level limit of detection values allow the measurement of $0.01 \mathrm{wt} \%$ loadings or less, depending on the elements (isotopes) to be detected. Almost all elements in the periodic system, with the exception of a few nonmetallic elements, can be measured. ${ }^{27}$ Nevertheless, ICP-MS requires the introduction of solution samples, therefore extensive chemical sample preparation (e.g. pressure or sonication assisted acid dissolution, etc.) is involved in the measurement. This makes the analysis time long (hours), especially if the dissolution procedure also needs to be developed specifically for the nanocomposites, and also increases the required solid nanocomposite sample amount to the mg level (an amount that can be accurately weighed and handled). The preparation and measurement of solution calibration samples is also needed, which again adds extra time to the analysis, but the quantitative results will also be more accurate this way. It has to be mentioned that solution-mode ICP-MS measurements are also prone to interference, the most severe here being the interference from precursor residues and dissolution reagent contaminants. These effects can give rise to a significant positive error in the result of the determination. In the present application, solution ICP-MS gave a Pt concentration result that is nearly three times higher than the TEM result, in spite of the ultra trace analytical conditions used. The precision was excellent, about $1 \%$ RSD.

Table 2 summarizes some features of the five analytical methods tested relevant in this application.

\section{Conclusions}

Five analytical techniques were tested in the application of the determination of the $\mathrm{Pt}$ concentration in a novel $\mathrm{Pt} / \mathrm{SiO}_{2}$ nanocomposite (Stöber silica particles decorated with ultra small, $1.6 \mathrm{~nm}$ diameter, Pt NPs). Four of these analytical methods (SEM-EDS, TEM imaging, XPS and solution-mode ICP-MS) are well established methods often used for the characterization of nanoparticles. The fifth method, single particle ICP-MS, is a novel, upcoming technique, which has not yet been tested earlier in the literature for nanocomposite characterization.

After performing repeated analyses on the as-synthesized nanocomposite and discussing the limitations of the accuracy of each method, we found that spICP-MS has an excellent potential in nanocomposite characterization. The spICP-MS analysis is fast; it requires only a small amount of sample, largely free from interference and gives accurate and precise results that agree well with the results from TEM imaging analysis. We would like to emphasize that a great benefit of using spICP-MS over the classical solution-mode ICP-MS for the quantitative analysis of nanodispersions is that spICP-MS can differentiate between the analyte contents present in ionic (dissolved) form and in particle form. This can help in avoiding large inaccuracies in quantitative NP analysis. We also successfully demonstrated, for the first time in the literature, that the cumulated spICP-MS signal from ultra small, individually not detectable NPs present on the surface of a nanocomposite allows accurate quantitation. Our results indicate that a careful choice of the measurement technique has to be made when determining the load concentration in nanocomposites.

\section{Acknowledgements}

This work was supported by the János Bolyai Research Scholarship of the Hungarian Academy of Sciences and the New 
National Excellence Program of the Ministry of Human Capacities (ÚNKP-ÚNKP-16-4) as well as the Hungarian Research Development and Innovation Office (NKFIH OTKA PD 120877) of András Sápi. Ildikó Kálomista is thankful for the financial support from the Campus Mundi program (EFOP-3.4.2-VEKOP15-2015-00001).

\section{References}

1 D. Madarász, G. Pótári, A. Sápi, B. László, C. Csudai, A. Oszkó, Á. Kukovecz, A. Erdőhelyi, Z. Kónya and J. Kiss, Phys. Chem. Chem. Phys., 2013, 15, 15917-15925.

2 H. Huang and D. Y. C. Leung, J. Catal., 2011, 280, 60-67.

3 A. Steinbrück, O. Stranik, A. Csaki and W. Fritzsche, Anal. Bioanal. Chem., 2011, 401, 1241-1249.

4 K. Z. Brainina, L. G. Galperin and E. V. Vikulova, J. Solid State Electrochem., 2012, 16, 2357-2363.

5 I. S. Park, O. H. Kim, J. W. Kim, Y. H. Cho and Y. E. Sung, J. Appl. Electrochem., 2016, 46, 77-83.

6 A. Sapi, F. Liu, X. Cai, C. M. Thompson, H. Wang, K. An, J. M. Krier and G. A. Somorjai, Nano Lett., 2014, 14, 67276730.

7 H. Wang, A. Sapi, C. M. Thompson, F. Liu, D. Zherebetskyy, J. M. Krier, L. M. Carl, X. Cai, L.-W. Wang and G. A. Somorjai, J. Am. Chem. Soc., 2014, 136, 10515-10520.

8 J. F. Gómez-Pérez, D. G. Dobó, K. L. Juhász and Z. Kónya, Catal. Today, 2017, 284, 37-43.

9 M. Czaun, J. Kothandaraman, A. Goeppert, B. Yang, S. Greenberg, R. B. May, G. A. Olah and G. K. S. Prakash, ACS Catal., 2016, 6, 7475-7484.

10 N. Halonen, A. Rautio, A.-R. Leino, T. Kyllönen, G. Tóth, J. Lappalainen, K. Kordás, M. Huuhtanen, R. L. Keiski, A. Sápi, M. Szabó, Á. Kukovecz, Z. Kónya, I. Kiricsi, P. M. Ajayan and R. Vajtai, ACS Nano, 2010, 4, 2003-2008.

11 D. Kim, J. Resasco, Y. Yu, A. M. Asiri and P. Yang, Nat. Commun., 2014, 5, 4948.

12 C. Degueldre and P.-Y. Favarger, Colloids Surf., A, 2003, 217, 137-142.

13 J. Liu, K. E. Murphy, R. I. MacCuspie and M. R. Winchester, Anal. Chem., 2014, 86, 3405-3414.
14 M. D. Montaño, J. W. Olesik, A. G. Barber, K. Challis and J. F. Ranville, Anal. Bioanal. Chem., 2016, 408, 5053-5074.

15 I. Kálomista, A. Kéri and G. Galbács, J. Anal. At. Spectrom., 2016, 31, 1112-1122.

16 F. Laborda, E. Bolea and J. Jiménez-Lamana, Anal. Chem., 2014, 86, 2270-2278.

17 J. W. Olesik and P. J. Gray, J. Anal. At. Spectrom., 2012, 27, 1143-1155.

18 F. Laborda, M. S. Jiménez, E. Bolea and J. Castillo, Int. J. Mass Spectrom., 2011, 307, 99-104.

19 S. Lee, X. Bi, R. B. Reed, J. F. Ranville, P. Herckes and P. Westerhoff, Environ. Sci. Technol., 2014, 48, 10291-10300.

20 H. E. Pace, N. J. Rogers, C. Jarolimek, V. A. Coleman, E. P. Gray, C. P. Higgins and J. F. Ranville, Environ. Sci. Technol., 2012, 46, 12272-12280.

21 R. J. Peters, Z. H. Rivera, G. van Bemmel, H. J. Marvin, S. Weigel and H. Bouwmeester, Anal. Bioanal. Chem., 2014, 16, 3875-3885.

22 W.-W. Lee and W.-T. Chan, J. Anal. At. Spectrom., 2015, 30, 1245-1254.

23 I. Kálomista, A. Kéri and G. Galbács, Optimization of plasma sampling depth and aerosol gas flow rates for single nanoparticle analysis by inductively coupled plasma mass spectrometry, Talanta, 2017, under review.

24 F. Laborda, J. J. Lamana, E. Bolea and J. R. Castillo, J. Anal. At. Spectrom., 2011, 26, 1362-1371.

25 L. C. Yung, C. C. Fei, J. S. Mandeep, N. Amin and K. W. Lai, J. Electron. Imaging, 2015, 24, 061105.

26 S. Amelinckx, D. van Dyck, J. van Landuyt and G. van Tendeloo, Electron microscopy: principles and fundamentals, ISBN 3-527-29479-1, Wiley-VCH, Germany, 1997.

27 F. Vanhaecke and P. Degryse, Isotopic Analysis Fundamentals and applications using ICP-MS, Wiley-VCH \& Co. KGaA, Weinheim, Germany, 2012.

28 W. Stöber and A. Fink, J. Colloid Interface Sci., 1968, 26, 6269.

29 N. C. Bigall and A. Eychmüller, Philos. Trans. R. Soc. London, 2010, 368, 1385-1404. 